\title{
Detection of Geometric Phases in Flux Qubits with Coherent Pulses
}

\author{
Z. H. Peng, M. J. Zhang, and D. N. Zheng*
}

National Laboratory for Superconductivity, Institute of Physics and Beijing National Laboratory for Condensed Matter Physics, Chinese Academy of Sciences, Beijing 100080, PR China

(Dated: November 10, 2018)

\begin{abstract}
We propose a feasible experimentally scheme to demonstrate the geometric phase in flux qubits by means of detuning coherent microwave pulse techniques. Through measuring the probability of the persistent current state in flux qubits, one can detect the Berry phase that is acquired with system's Hamiltonian adiabatical circular evolution in the parameter space. Furthermore, we show that one could choose an appropriate amplitude of pulses in an experiment to obtain high readout resolution when detuning frequency of pulses is fixed and controlled phase shift gates can be implemented based on the geometric phases by inductance coupling two flux qubits.
\end{abstract}

PACS numbers: Valid PACS appear here

The unrivaled computational power of quantum computers in solving certain problems that are virtually impossible for classical computers has made quantum computing one of the hottest topics in current research [1]. The building blocks of quantum computer are called quantum bits or qubits. A variety of different physical systems have been explored to implement qubits. For constructing a practical quantum computer, solid state qubits with scability are believed to be very important. So far, superconducting qubits based on Josephson junctions have emerged as a very promising candidate of solid state qubits 2, 3, 4]. Up to now, not only single superconducting qubits have been realized but also entanglement between two coupled qubits has been observed experimentally [5, 6, 7]. Superconducting qubits can be approximately divided into three categories, i.e. charge qubit, flux qubit and phase qubit. Furthermore, superconducting qubits, considered as artifical macroscopic two-level atoms, are used to test fundamental laws in quantum mechanics [5, 8, 9, 10].

When a quantum-mechanical system undergoes an adiabatic cyclic evolution, it acquires a geometric phase factor, called Berry's phase, in addition to the dynamical one 11. This effect has been observed in many microscopic systems 12]. Based on the geometric phase, one can realize any quantum logic operations which can be composed from nontrivial two-qubit gates (such as a controlled phase shift gate) and single-qubit gates 13, 14]. Compared with conventional quantum computation, the geometric quantum computation approach offers the potential of fault-tolerance. The Berry phase does not depend on the details of the motion along the path in parameter space but only depends on the area enclosed by the system's parameters over a cyclic evolution in parameter space. Therefore, the Berry phase is left unchanged by an imperfection on the path as long as the area is left unchanged. Because of this feature, it has been suggested that the Berry phase could be a useful tool for intrinsically fault-tolerant quantum computation [15, 16, 17, 18]. Conditional Berry phase gates have been demonstrated in NMR and in trapped ions [15, 16]. This is at the heart of geometric quantum computation.

However, it is not clear whether the effect exists in macroscopic quantum systems. Falci et al. 17] have proposed a scheme to observe the Berry phase and use the Berry phase to design universal quantum logic gates in charge qubits. There are many other schemes that have been proposed to detect geometric phase or realize geometric quantum computation in superconducting qubits 14, 19]. However, almost all of these schemes are based on charge qubits. In this letter, we show that these two objects can also be realized in flux qubits with coherent pulse techniques [20]. We believe that due to the long decoherence time and relatively simple device fabrication process it may be easier to observe the Berry phase in flux qubits than in charge qubits [2, 4, 17]. Especially, dephasing time of 4 Josephson junctions flux qubits have achieved $1 \mu s$ [21].

Coherent pulse sequence technique.-NMR techniques are often used in coherent control quantum systems now being considered for the implementation of quantum computer 22]. Collin et al. 23. have demonstrated NMR-like control of a qubit superconducting circuit with multipulse sequences. More recently, Kutsuzawa et al. 24] have observed Ramsey fringes by applying a pair of phase-shifted microwave pulses without introducing detuning. A clear advantage the method over conventional detuning method is that it provides much faster operation. The coherent pulse technique is one of the techniques widely used in NMR [20]. The oscillating radio-frequency field produced in a coil by a sequence of pulses occurring at times $t_{1}, t_{2}, \ldots, t_{k}$ and of durations $\tau_{1}, \tau_{2}, \ldots, \tau_{k}$ can be represented by the function

$$
V=\sum_{k} V_{k}(t) \cos \left(\omega_{r f} t+\varphi_{k}\right),
$$

where $V_{k}(t)=0$ outside of the interval $t_{k} \leq t \leq t_{k}+\tau_{k}$ and is approximately constant inside. The pulses are called incoherent if the phases $\varphi_{k}$ are distributed at random, and coherent if their values can be controlled. The orientation of the rotating field with respect to the rotating frame varies at random between pulses in the first 


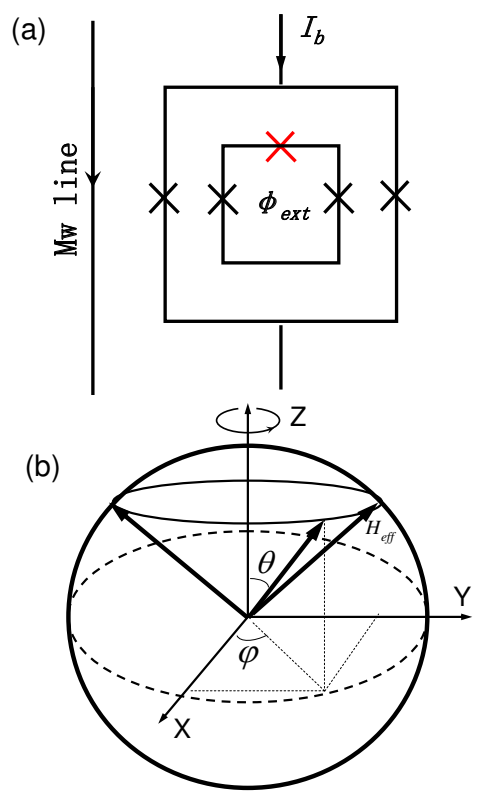

FIG. 1: Schematic design of the system. (a) The flux qubit, pierced by a magnetic flux $\phi_{\text {ext }}$, and microwaves(MW) are applied to the qubit from the on-chip strip line. The quantum state of the flux qubit can be read out by the switching probability of the biased DC-SQUID. (b) Evolution of the effective Hamiltonian in parameter space by adiabatically varying the phase of the radio frequency. Each pulse makes the Hamiltonian rotate about the $z$-axis at a fixed angle $\varphi$. After the circular evolution, the Berry phase acquired in flux qubits is $\pm \pi(1-\cos \theta)$, where $\varphi$ is the phase of the MW pulse and $\theta=\arctan \frac{\nu \sin \eta}{\Delta \omega}$ is the cone angle.

method, but is well defined in the second( in particular, can be made fixed ). Coherent pulses have another advantage besides controlling the phase of the rotating field: the signal-to-noise ratio is considerably improved. Considering flux qubits, a circular motion of the system Hamiltonian can be imposed by adiabatically varying the phase of the radio frequency.

Flux qubit and the rotational framework. - We first consider a single flux qubit and the measurement circuit is described in Ref. [24, 25]. The relative phase shift between these pulse sequences is precisely controlled by synchronous microwave generators [20, 24].

A flux qubit can be described as a two-state system or pseudo spin $1 / 2$ particle [26]. In terms of the Pauli spin matrices $\hat{\sigma}_{z}$ and $\hat{\sigma}_{x}$, we can write the Hamiltonian as $\hat{H}_{0}=\frac{\hbar}{2}\left(\varepsilon_{f} \hat{\sigma}_{z}+\Delta \hat{\sigma}_{x}\right)$, where $\Delta$ is tunnel splitting, $\varepsilon_{f} \approx I_{p}\left(\phi_{\text {ext }}-1 / 2 \phi_{0}\right)$ is the dc energy bias $\left(I_{p}\right.$ is the circulating current of a flux qubit). The two eigenstates of $\hat{\sigma}_{z}$ are macroscopically distinct states with the qubit persistent current circulating in opposite directions, i.e., the clockwise state $|1\rangle$ and the counterclockwise state $|0\rangle$. We employ a microwave current burst to control the qubit state and induce oscillating magnetic fields in the qubit loop. That event is described by the perturbation Hamiltonian $\hat{V}=\frac{\hbar}{2} V(t) \hat{\sigma}_{z}$, where $V(t)=2 \nu \cos \left(\omega_{r f} t+\varphi\right)$ has an amplitude correlated to the power of the applied microwave pulses. The total
Hamiltonian can be transformed by rotating the matrix according to $U(\eta)\left(\hat{H}_{0}+\hat{V}\right) U^{-1}(\eta)$, where $U$ contains the normalized eigenvectors of the Hamiltonian in the flux basis 27],

$$
U(\eta)=\left(\begin{array}{cc}
\cos \frac{\eta}{2} & -\sin \frac{\eta}{2} \\
\sin \frac{\eta}{2} & \cos \frac{\eta}{2}
\end{array}\right)
$$

where $\eta=\arctan \left(\Delta / \varepsilon_{f}\right)$. We obtain the total Hamiltonian $\hat{H}=-\frac{\hbar}{2}\left\{\left[\omega_{0}-\frac{\varepsilon_{f}}{\omega_{0}} V(t)\right] \hat{\sigma}_{z}+\frac{\Delta}{\omega_{0}} V(t) \hat{\sigma}_{x}\right\}$, where $\omega_{0}=\sqrt{\varepsilon_{f}^{2}+\Delta^{2}}$ is the qubit Larmor frequency at the measured flux bias point. However, we can disregard $\frac{\varepsilon_{f}}{\omega_{0}} V(t)$ because under the usual experimental conditions, $\frac{\varepsilon_{f}}{\omega_{0}} \nu<\omega_{0}[10]$. This is analogous to the spin $1 / 2$ particle, and because the Berry phase is gauge-invariant, we write the Hamiltonian in the rotating frame approxiamation [20], $\hat{H}_{\text {eff }}=\left(\hbar \omega_{r f} / 2\right) \hat{\sigma}_{z}+e^{i\left(\omega_{r f} t / 2\right) \hat{\sigma}_{z}} \hat{H} e^{-i\left(\omega_{r f} t / 2\right) \hat{\sigma}_{z}}$ and obtain

$$
\hat{H}_{\mathrm{eff}}=\frac{\hbar}{2}\left(\begin{array}{cc}
\omega_{r f}-\omega_{0} & -\nu e^{i \varphi} \sin \eta \\
-\nu e^{-i \varphi} \sin \eta & \omega_{0}-\omega_{r f}
\end{array}\right)
$$

The effective Hamiltonian is read $\hat{H}_{\text {eff }}=$ $-(1 / 2) B \cdot \hat{\sigma}$, so we have defined fictitious field $B \equiv \hbar(\nu \cos \varphi \sin \eta, \nu \sin \varphi \sin \eta, \Delta \omega)$, where $\Delta \omega=\omega_{0}-\omega_{r f}$. The flux qubit thus behaves like a spin $1 / 2$ particle in a magnetic field. Under the action of coherent pulse sequences, the effective qubit Hamiltonian describes a cylindroid in the parameter space $\{B\}$.

Creating the Berry phase in a single flux qubit.Analogous to a spin $1 / 2$ particle, the Hamiltonian lies close to the $z$-axis when $|\nu| \ll|\Delta \omega|$, while the Hamiltonian lies close to the $x-y$ plane when $|\nu| \gg|\Delta \omega|$. If the applied radio-frequency radiation is far from resonance, the Hamiltonian is quantized along the $z$-axis, and if the radio frequency is swept towards resonance $(\Delta \omega=0)$, the Hamiltonian rotates from the $\mathrm{z}$-axis towards the $x-y$ plane. If the resonance is approached sufficiently slowly, the spin will follow the Hamiltonian in the rotating frame according to the adiabatic theorem. Next, by adiabatically varying the phase of the radio frequency, a circular motion can be performed. When the Hamiltonian returns to the $x-y$ plane the frequency sweep may be reversed, so that the spin returns to its original state, aligned along the $z$-axis. The Berry phase acquired in this cyclic process is $\pm \pi$, where the signs \pm depend on whether the system is in the eigenstate aligned with or against the Hamiltonian. If we don't sweep the radio-frequency all the way to resonance, but only to some final value $\omega_{r f}$, the Hamiltonian ends at some angle to the $z$-axis, and so flux qubit with arbitrary cone angles can be realized. A similar case occurs if one replaces the frequency sweep with an amplitude sweep, in which the radio frequency is always applied in off-resonant conditions, and its amplitude is raised from zero to some final value, $\nu$.

We now describe a procedure to measure the Berry phase in experiments. First, the flux qubit is prepared 


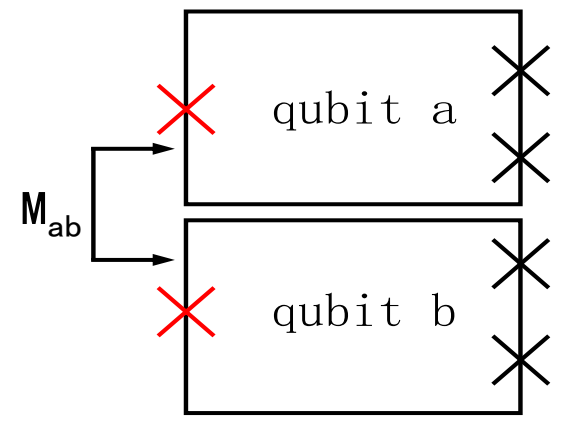

FIG. 2: Schematic design of the two-bit gate. The two qubits can be coupled via their mutual inductance. The Berry phase of the target qubit (qubit a) can be controlled by the control qubit (qubit b).

in the ground state of the Hamiltonian at the degeneracy point and then a hard pulse is applied to excite the flux qubit in a linear superposition of the energy eigenstates which is analogous to the splitting of the photon wavefunction at the first beamsplitter of a Mach-Zender interferometer. Next, appropriate amplitude adiabatic coherent pulse sequences are applied. With the geometric phases acquired in this way, we will have an additional dynamical component, which depends on experimental details. This can be eliminated using a conventional spin echo approach: the pulse sequence is applied twice, with the second application surrounded by a pair of $180^{\circ}$ pulses applied to the spin particle. The final step in the procedure is to measure the persistent current state of the qubit. The probability of measuring the persistent current state $|1\rangle$ in the flux qubit at the end of this procedure is given by

$$
P(1)=\sin ^{2}(2 \gamma),
$$

independent of the elapsed time, where $\gamma$ is the Berry phase that is acquired in the first adiabatic loop process. If the initial state of the flux qubit is not prepared at the degeneracy point, we have to deduce the Berry phase value $\gamma$ from the interference pattern of the persistent current state $|1\rangle$ measuring probability.

For the quantum mechanics system, the acquired geometric phase is equal to half of the solid angle subtended by the area in the parameter space enclosed by the closed evolution loop of fictitious magnetic field,

$$
\gamma=\pi\left(1-\frac{\Delta \omega}{\sqrt{\Delta \omega^{2}+(\nu \sin \eta)^{2}}}\right)
$$

If we fix the bias point at the degeneracy point, $\sin \eta=1$, geometric phases to achieve a NOT gate for $\nu=\sqrt{3} \Delta \omega$ and Hadamard gate for $\nu=\frac{\sqrt{7}}{3} \Delta \omega$.

The adiabatic conditional geometric phase gate.-For the implementation of a universal two-qubit gate, we consider in a system of two coupled flux qubits with their mutual inductance. The two qubits are now described
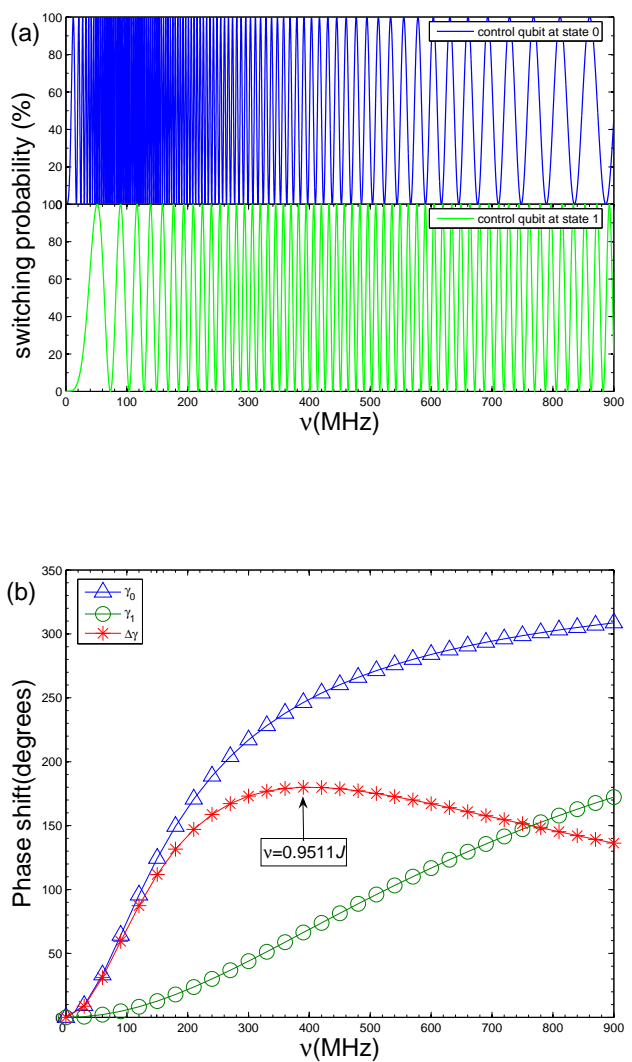

FIG. 3: Calculated results of the controlled phase shift in the target qubit. Here, the pulse sequence is applied twice in order to eliminate the dynamics phase. The split energy of the two coupled flux qubits is chosen from Ref.[5], $J=420 \mathrm{MHz}, \Delta \omega=129.78 \mathrm{MHz}$. (a) The switching probability of the persistent current state $|1\rangle$ in target qubit (qubit a) vs. amplitude of coherent pulses. At low amplitude region, the switching probability of the target qubit when the control qubit at state $|0\rangle$ oscillates very quickly. Therefore, one should choose an appropriate amplitude of pulses in an experiment to obtain high readout resolution. (b) Berry phase $\gamma_{0}, \gamma_{1}$ and the controlled Berry phase difference $\Delta \gamma$ as a function of the amplitude of microwave pulse. Open triangles, circles and stars denote $\gamma_{1}, \gamma_{0}$ and $\Delta \gamma$ respectively. Near the $\nu=0.9511 J$, controlled phase shift dependence on the $\Delta \omega$ is reduced to second order.

by the total Hamiltonian:

$$
\hat{H}=\sum_{i=a, b} \hat{H}_{i}+J \hat{\sigma}_{z}^{(a)} \hat{\sigma}_{z}^{(b)}
$$

Two qubits can be coupled via the magnetic inductance or a large Josephson junction for different coupled strength 28].

We consider that the $\Delta$ of the two qubits is different and the Larmor frequency of the two qubits is different at the degeneracy point, and only qubit $a$ is close to resonance. In practice, the two qubits usually have different parameter values because of fabrication limitations 29]. The two transitions of qubit a (corresponding the two current states of qubit $b$ ) will be split by $\pm \pi J$, and so will have different resonance offsets. With the coherent radio-frequency microwave pulses, the effective Hamiltonian depends on the resonance offset, and so the cone angle (and hence the Berry phase acquired) will depend 
on the state of qubit a. This permits a conditional Berry phase to be applied to qubit a, where the size of the phase shift is controlled by qubit $b$. If the frequency is applied at a frequency $\Delta \omega$ away from the resonance frequency of qubit $a$ when qubit $b$ (the control qubit) is in state 0 , and $\nu$ is the amplitude of radio-frequency pulses, then the differential Berry phase shift

$\Delta \gamma= \pm \pi\left(\frac{\Delta \omega+J}{\sqrt{(\Delta \omega+J)^{2}+(\nu \sin \eta)^{2}}}-\frac{\Delta \omega}{\sqrt{\Delta \omega^{2}+(\nu \sin \eta)^{2}}}\right)$

depends only on $\Delta \omega, \nu$ and $J$; it is independent of how the process is carried out as it is slow enough to be adiabatic, but rapid compared with the decoherence times. A range of controlled Berry Phases can be obtained by choosing appropriate values of $\Delta \omega$ and $\nu$ ( $J$ is fixed by the two coupled flux qubit with mutual inductance.) If we fix the value of $\Delta \omega / J$, the controlled Berry phase will rise and then fall as $\nu$ is increased. We can make the approach very robust when the desired $\Delta \gamma$ occurs at the maximum in this curve. Then the dependence on the $\Delta \omega$ is reduced to second order 15. To achieve the controlled-NOT gate, we fix the bias point at the degeneracy point $\sin \eta=1$ and we can design a controlled $\pi$ shift at $\Delta \omega=0.309 \mathrm{~J}$ and $\nu=0.9511 J$, as showed in Fig B

We desicribe an example to illustrate the detection of the Berry phase in a single flux qubit by applying coherent pulse sequences. The parameters were as follows, split energy $\Delta=6 \mathrm{GHz}$, detuning frequency $\Delta \omega=150 \mathrm{MHz}$, phase sweeps implemented using 100 linear steps of about $3 \mathrm{~ns}$, giving a total pulse sequence length of about $600 \mathrm{~ns}$. These parameters satisfy the adiabatic threshold $\frac{\nu}{T_{2}} \ll$ $\frac{d H_{e f f}}{d t} \ll \nu^{2}[20]$, where $T_{2}$ is the dephasing time of the flux qubit. The required adiabatic manipulation time not longer than current flux qubits' performance and the required coherent pulse techniques is mature 20, 21, 22]. These indicate we can detect the geometric phase in a flux qubit with coherent pulses in experiments.

Conclusion.-We have proposed a new experimental scheme to detect geometric phases with coherent microwave pulses in flux qubits. Through measuring the persistent current state in the flux qubit, we can detect the Berry phase which is acquired in circular motion. Furthermore, we have designed one-bit quantum logic gate and two-bit controlled phase shift gate based on the Berry phase. The idea of the geometric phase shift gate demonstrated by the flux qubits system here should in principle also work for other superconducting qubits.

We thank P. L. Lang, A. Maassen van den Brink, C. P. Sun, W. M. Liu and A. Ahmad for useful discussions and J. Q . You for useful help.This work is supported by National Nature Science Foundation of China(10221002), Ministry of Science and Technology of China through the 973 program(2006CB601007) and Chinese Academy of
Sciences.

* electronic address: dzheng@ssc.iphy.ac.cn

[1] M. A. Nielsen and I. L. Chuang, Quantum Computation and Quantum Information (Cambridge University Press, Cambridge, 2000).

[2] Yu. Makhlin, G. Schön and A. Shnirman, Rev. Mod. Phys. 73, 357 (2001).

[3] Y. Nakamura, Yu.A. Pashkin, and J. S. Tsai, Nature (London) 398, 786 (1999); J. R. Friedman et al., ibid. 406, 43 (2000); Y. Yu et al., Science 296, 889 (2002); D. Vion et al., Science 296, 886 (2002); J. M. Martinis, S. Nam, J. Aumentado, and C. Urbina, Phys. Rev. Lett. 89, 117901 (2002).

[4] J. E. Mooij et al., Science 285, 1036 (1999).

[5] A. Izmalkov et al., Phys. Rev. Lett. 93, 037003 (2004).

[6] Y. A. Pashkin et al., Nature(London) 421, 823 (2003); T. Yamamoto et al., ibid. 425, 941 (2003).

[7] A. J. Berkley et al., Science 300, 1548 (2003).

[8] Y. Yu et al., Phys. Rev. Lett. 92, 117904 (2004).

[9] A. J. Leggett, J. Phys. Condens. Matter 14, R415(2002); A. Wallraff et al., Nature (London) 431, 162 (2004); I. Chiorescu et al., ibid. 431, 159 (2004).

[10] S. Saito et al., Phys. Rev. Lett. 93, 037001 (2004).

[11] M. V. Berry, Proc. R. Soc. Lond. A 392, 45 (1984).

[12] A. Tomita and R. Y. Chiao, Phys. Rev. Lett. 57, 933 (1986); R. Tycko, ibid. 58, 2281 (1987); T. Bitter and D. Dubbers, ibid. 59, 251 (1987); R. Tycho, ibid. 58, 2281 (1987).

[13] S. Lloyd, Phys. Rev. Lett. 75, 346 (1995).

[14] S. L. Zhu and Z. D. Wang, Phys. Rev. Lett. 89, 097902 (2002).

[15] J. Jones, V. Vedral, A. Ekert and G. Castagnoli, Nature (London) 403, 869 (2000).

[16] D. Lelbfrled et al., Nature (London) 422, 412 (2003).

[17] G. Falci et al., Nature (London) 407, 355 (2000).

[18] L. M. Duan, J. I. Cirac and P. Zoller, Science 292, 1695 (2001).

[19] S. L. Zhu, Z. D. Wang and P. Zanardi, Phys. Rev. Lett. 94, 100502 (2005); X. B. Wang and M. Keiji, Phys. Rev. B 65, 172508 (2002); A. Blais and A. M. Tremblay, Phys. Rev. A67, 012308 (2003); P. Zhang et al., ibid. 71, 042301 (2005).

[20] A. Abragam, The Principles of Nuclear Magnetism ( Clarendon, Oxford, 1961).

[21] Y. Nakamura et al. (unpublished).

[22] L. M. K. Vandersypen and I. L. Chuang, Rev. Mod. Phys. 76, 1037 (2004).

[23] E. Collin et al., Phys. Rev. Lett. 93, 157005 (2004).

[24] T. Kutsuzawa et al., Appl. Phys. Lett. 87, 073501 (2005).

[25] I. Chiorescu, Y. Nakamura, C. J. P. Harmans and J. E. Mooij, Science 299, 1869 (2003).

[26] T. P. Orlando et al., Phys. Rev. B 60, 15398 (1999).

[27] M. C. Goorden, Master's thesis, Technical University of Delft, Delft, 2002.

[28] A. C. J. Ter Haar, PhD's thesis, Technical University of Delft, Delft, 2005.

[29] J. B. Majer et al., Phys. Rev. Lett. 94, 090501 (2005). 